\title{
Exploring the complexity of understanding, managing and marketing codes for sustainability in the current economic climate - issues for the small builder
}

Erik Geelhoed, Andy Morrell, Praminda Caleb-Solly and Ming Sun

University of the West of England, Faculty of Environment and Technology

Frenchay Campus, Bristol BS16 1QY, UK

Email: Erik.Geelhoed@uwe.ac.uk

Keywords: Code for Sustainable Homes (UK), Small to Medium Enterprise Builders, user and attitude research, technology use.

In the UK the Code for Sustainable Homes (CSH) is set to become mandatory in 2016. However current attitudinal research highlights unwillingness within the major construction industry although there is some promising research regarding occupier awareness. Little is known about attitudes of Small to Medium Building Enterprises regarding the $\mathrm{CSH}$. We conducted an in-depth interview study with builders and found that by and large they have a good understanding of the CSH and value a good relationship with building inspectors. In addition they are not computer illiterate and have been using technology for decades. Their use of "estimating" software allows them to gain intricate knowledge of all the different stages of the building process and as such presents itself as a promising vehicle to also educate them about the CSH.

\section{Introduction}

The UK based Concepts to Completion Design Tools for Sustainable Buildings program is funded by the Technology Strategy Board (TSB) in collaboration with HBXL [WEB ref 1] and the University of the West of England (UWE) in Bristol. HBXL is a Bristol based company, which has been selling award winning software aimed at Small to Medium Building Enterprises (SME) and have over 9000 users. The aim is to develop an easy to use integrated tool for SMEs to design buildings from initial concepts and feasibility through detailed design for low impact buildings in the UK.

It has been reported [5] that the building construction industry uses up a large amount of global resources and is responsible for approximately $40 \%$ of the total global energy consumption; during the construction phase in the form of embodied energy and during the operation phase as operating energy. Even though, current interpretations of embodied energy are quite unclear and vary greatly and there may be no reliable template, standard or protocol regarding embodied energy [5], where global warming is concerned, reducing energy consumption and carbon emission in building and occupying houses is paramount.

In most of the UK (although not in Scotland), the main method of enforcing higher energy performance of buildings has been through building regulations and the Code for Sustainable Homes (CSH), the environmental impact 
rating system for newly built housing. The CSH sets six levels of sustainability for new build housing ("Level 6" being the highest) against nine design categories, rating the "whole home" as a complete package (e.g. CO2 Emissions, Water, Materials, Waste) and is set to become mandatory in 2016 [1].

The UK construction industry as a whole has an annual turnover of more than $£ 100$ billion and accounts for almost 10\% of the country's GDP [WEB ref 5]. Within this sector there are about 37,000 registered SMEs building companies, roughly $15 \%$ of the total construction industry. With regards to the viability of implementing the CSH by 2016 it is important to evaluate attitudes within the construction industry.

Osmani \& O'Reilly [10] gauge the feasibility of building zero carbon homes by 2016 from the perspective of major UK housing developers. The authors list numerous legislative, cultural, financial and technical barriers facing house builders. Their attitudinal survey resulted in 41 returned postal questionnaires filled out by major house builders. As a follow up, eight high level managers from the corporate world of house builders were interviewed.

The survey questions were in the form of 5-point Likert scales [WEB ref 4]. In a typical five-point Likert scale question, a participant is asked to select what they feel reflects their feelings, attitudes etc. best, ranging from 1.Strongly disagree, 2.Disagree, 3.Neither agree nor disagree, 4.Agree to 5.Strongly agree.

The statistical analysis of Osmani's questionnaire is minimal and doesn't go any further than listing percentages, but this is still revealing, e.g. $51 \%$ of respondents comment that they have a sustainability policy in place and a further $29 \%$ stating that they were in the process of creating one. Similarly, $56 \%$ of the house builders surveyed reported that they have a Corporate Social Responsibility (CSR) policy or were in the process of developing one. However, only $7 \%$ of house builders hold the environmental management ISO 14001 accreditation [WEB ref 3], which, the authors state, seems rather contradictory.

Because of mandatory requirements of using high efficiency condensing boilers, the construction industry has been adopting a consistent approach in implementing thermally efficient and low air permeability building fabric. This contrasts with the frequency of using high efficiency glazing, which is commonly used by only $5 \%$ of respondents. Equally, just $12 \%$ said that they always integrate renewable energy features in their housing projects. Customer demand is seen as an essential element in the widespread development of zero carbon homes. In addition they put the responsibility for existing barriers to the adoption of the $\mathrm{CSH}$ on legislation and the supply chain.

This account of dependency on consumers, legislation and supply chain contrasts sharply with the proactive stance of the Turner Construction Company, one of the largest construction management companies in the United States. Deane [3], an employee of Turner, has a deep understanding of the merciless competitive environment of the construction industry. From this informed position he observes, using projects completed by the Turner Construction Company as examples, that there is a high degree of misperception on the part of decision makers about the true costs and benefits of green buildings that have slowed the adoption of green buildings in the commercial market.

Following guidelines of the Leadership in Energy and Environmental Design (LEED) V1.0 in 2000, Turner began work on a 42-storey high-rise building in New Jersey, which, in 2000, was perceived to be an expensive and risky proposition. The risk seems to have paid off as by 2007, the development 
community, clearly recognized that tenants were interested in LEED-certified buildings and, indeed, demanded them. This was, in part, because of operating cost savings, but also because of the perception that these buildings were healthier environments in which to live and work, and would result in a more productive workforce, reduced absenteeism and reduced incidence of illness. Although the perception was that this would add $15-18 \%$ to the cost, Dean estimated that the true cost premium for green buildings is closer to $2 \%$. Thus encouragingly, powerful sections of the design and construction community (in the US at least) are striving to create "net zero energy" buildings.

It is now increasingly recognised that technological solutions to domestic energy reductions are insufficient and cannot guarantee energy savings without the cooperation of the occupants. Energy use in identical homes with different occupants can vary by a factor of three $[8,7]$. Both sets of authors recommend the use of the Post Occupancy Evaluation (POE) questionnaire, a tool to evaluate whether there is a discrepancy between designers and users (occupants). The key parameters for which occupant feedback is collected encompasses perception of comfort (temperature, daylight, noise, heating), satisfaction (space, best and worst aspects of the house), behaviour (how heating and hot water are used, use of appliances) and expectations about refurbishment. However, it is not entirely clear from the reports, what it is that the authors found using the POE.

Gill [7] asked occupants to estimate their heat, electricity and water consumption and claim they found considerable differences with the actual measured (heat, electricity and water) use. However, the evidence does not quite stack up. Analysing Likert scale responses using Pierson's product moment correlation, it was found that there was a highly positive correlation of $r=0.83$ (the authors prefer the $\mathrm{R}^{2}$ version, which suggests a much more tenuous relation) between estimated and actual measured heat consumption. Unfortunately, degrees of freedom and level of significance were not shown. Similarly electricity consumption seems to be estimated reasonably well, $r=0.62$. However, water consumption, still a respectable $r=0.54$, did result in a less accurate estimate; but whether this was or was not statistically significant has been omitted by the authors.

The UK Green Building Council [6] carried out extensive research on understanding consumer attitudes to "Sustainable Community Infrastructure". The survey, comprising of over 1000 people and again using five point Likert scales, evaluated attitudes to (hypothetical) communal sustainable building and infrastructure methods, where communities would share the burden of a green infrastructure with (local) authorities.

The results are a mixture of a positive attitude towards sustainable techniques per se and concerns about the actual implementation of such a policy, in particular about what would happen if the infrastructure broke down and how to exclude "free-loaders" from benefitting. Some of these positive and negative attitudes were used to feed into a consumer segmentation model. There seems to be an even (33\%) split between three groups: on the positive side a group of "early adopters" and "energy source concerned" participants, on the negative end a group of "waste doubters" (sceptics) and then there is a poorly defined group which takes up the middle ground.

Ornetzedera and Rohracherb [9] evaluate whether user participation might be brought into play in the design process as a strategy to improve the development and dissemination of sustainable energy technologies. Reviewing 
case studies, and going back as far as the mid nineties of the last century, in the field of solar collectors and biomass heating systems as well as sustainable building technologies they argue that, in the case of renewable energy technologies, self-building groups were highly successful and this resulted in improved (as well as widely disseminated) technologies. The authors highlight an often cited program in Freiburg (Germany) where a residential area was developed. Five thousand (future) inhabitants organised themselves in groups of 5-15 families in order to plan and build together. In most cases, these groups were formed around existing social relations and were bound together by contracts, acquired the building ground, assigned an architect etc. and finally commissioned a construction company to realise the project. Many co-building groups brought about special ecological and social measures within their new homes beyond the official planning which already included low energy building, public transport and keeping cars outside the district. Most individuals were intensely involved in the planning process and in this way provided an unusual basis for technical innovation.

Per Christiansson and colleagues' [2] approach to "User Driven Innovation in the Building Process" is more systematic. They provide a clearly written overview of the benefits of user involvement, covering interviews, focus groups, questionnaires and a host of design oriented methods to elicit user requirements, as well as a first description of how to distil metrics. Although this paper looks promising, it does not include the results of actual data collection.

Little is known about attitudes of SME's in the building industry. SME builders are in a unique position in that they have direct contact (and on a daily basis with) with the customers. Thus, having their ears to the ground might inform us about current customer demand with regards to the uptake of sustainable building methods. Thus, it becomes desirable to understand the barriers to CSH adoption, whether there are specific training needs, or, conversely, whether there are facilitating factors. This forms the focus of the current paper.

In the interviews we evaluated the use of two HBXL software packages: the first one helps builders to estimate building projects (EstimatorXpress) and the second one is an easy to use Computer Aided Design (CAD) package (PlansXpress). We will present the results of this evaluation as a separate paper in future. However we will mention the use of the extensive data base that underlies the estimating package and how it might play an educational role in learning about the $\mathrm{CSH}$, as one of our research questions addresses whether there are opportunities within the software suite to educate builders around sustainable building methods.

\section{Method}

\subsection{Participants}

Eight participants were interviewed. Their job-profiles cover a range of activities: builders, homebuilders, architectural technologists, code assessors and quantity surveyors and mostly they have a small number of permanent employees. All the interviewees were male, although important feedback on lay-out of printed output was generated by wives who are often their business partners. Participants were not asked about their age, but all of them had been in the business for a considerable length of time, often for several decades. 


\subsection{Interview structure, data capture and analysis}

Participants were interviewed in their own office environment about their: general background; knowledge and use of sustainable building methods; general software use; mobile technologies and use of HBXL's software suite.

For the Use of HBXL's software suite the participants were asked to perform a cognitive walkthrough [4], a method whereby participants are asked to "think aloud" as they carry out computer tasks, of a typical use of EstimatorXpress and or PlansXpress whilst their computer screen was videoed.

Interviews were audio recorded and transcribed. The footage of real-life examples of their use of HBXL software resulted in video clips highlighting important aspects of where users encountered problems with the software or which aspects particularly met their needs.

\section{Results}

The participants in this study are from diverse backgrounds and although people's personal motivations for their career choices are different all have considerable (i.e. several decades) experience in their trade.

"I have been doing this ever since I left school back in 1987. I am a third generation carpenter, so I have been kicking around building sites summer holidays."

Interestingly someone's business expansion coincides with him acquiring EstimatorXpress.

"I've been in business since '89, I only recently started doing the general building stuff, I was a plumbing and heating engineer before and funny enough it coincides with when I got the software, that's when I started taking on different projects."

Most of them carry out a range of work and often they have more than one project on the go, even if they try not to.

"I just made someone a new toilet door for the outhouse right up to, eh, I just priced up this morning and won the contract for building a six bed-roomed house out of Beco. I have got an enquiry at the moment for 37 houses to be built and they want me as a main contractor."

The nature of the business is truly SME, ranging from a modest number of "permanent" employees to only one or even none. Taking on contractors as and when is needed is general practice. These contractors originate from small and trusted networks.

"I'll do anything, I'll go and change a lock, I build an extension.

Electrical work I tend to get a sub-contractor in; I will do small plumbing jobs but anything too major and I get a contractor in. [more than one job on the go?] I try not to. If it's small jobs, then, I might have a couple of jobs running. Very often you get a job where you can't finish it in one stage, you gotta and go and do so much and then someone else is coming in, so I will fit a smaller job in, in between."

There is a mixed response when asked whether recession has affected their business. Some are positive that it hasn't much.

"It hasn't affected me up until now. As I said I'm busy at the moment but I usually always used to be booked probably six months in advance, now we're down to sort of two or three months so there is a difference there." 
Whilst others were hit harder and state that they have to work harder for less.

"Well yes it's been difficult, we've had to work harder to do it; we're not making loads of money, like anybody else we're just turning the books over. We've been lucky in the fact that we've got some clients that we've worked for for years."

When questioned about whether they heard or knew of the Code for Sustainable Homes (CSH), there were those who had a copy of the guidelines on their desks.

"I'm very familiar with it. I have it; it's a permanent thing on my desk. --. Levels 5 and 6, oh yes I know about my code."

"I'm an Architectural Technician. I've been interested in green building and green architecture since 1980 when I graduated. So a few years ago I decided I've got to do something about it."

Whilst on the other end of the continuum one builder, had never heard of it. Not surprisingly those on that end of the spectrum also saw very little (economic or otherwise) sense in it; they just do what the customer asks for but most importantly also what the building inspector requires.

"I've heard things about it but to be honest with you I don't know much about it. I mean what do you class as Sustainable Homes, are you talking reusable energy and that sort of thing are you? A customer will come to me with a set of plans normally and I do what the customer asks or what Building Control requires."

More alarming is that recession might have a negative effect on the implementation of the code.

"I also think that if we were talking boom times, may be that would be a better time in terms of implementing code, whereas now they're looking at every penny. There are building sites at the moment where they bought the land at the peak three or four years ago and are trying to come out of this quite close to breaking even, they have to free up cash flow."

However, there are those for whom it actually is at the core of their business: using sustainable materials, actually being a Code Assessor and marketing sustainable houses. For this last person using 3D graphics proves to be an essential tool to show clients "around" in an eco-dwelling, using a combination of Skype and his website.

"An eco house design that I'm developing which I will be marketing on a website. The website is aimed at self-builders and they can choose how the house looks from the outside by choosing different materials for the ground floor, the first floor and the roof and also things like the level of construction so the basic level of construction is to Code Level 4. I also use Skype and what it can do is I can show people my screen and then I can turn it round and say this is what it's going to look from this side and so on and they can see it on their computer."

But even those who have poor knowledge of the $\mathrm{CSH}$, have come across green technologies. There is a general perception that sustainable materials and methods initially will push the price up which might scare clients off, even though in the long term the clients would benefit through e.g. lower heating bills.

"We did a partial loft conversion recently and the customer asked to have a green installation and all different types of green stuff but when they got the price they went back to the normal insulation and stuff because it was sort of three times more expensive. Another thing where I'm noticing the difference because obviously my background being plumbing and heating, obviously all the 
boilers now have got the condensers so that's something we've done that's green, we always get the condenser boilers."

Given the participants' perception that the building industry is not exactly pushing for sustainable building methods, do they believe there is more of a drive for change coming from the Government? The (previous) Government Legislation is perceived as a driving force towards a more sustainable future issuing corresponding legislation, although they regret that existing housing stock is not targeted.

"Well actually the last Labour Government did a huge thing which was introduce feed-in tariffs and also the renewable heating tariff which is the first one in the world and both of those have been very significant for pushing low carbon technology adoption in this country. I just hope the current Conservative Government doesn't cut it back in any way, it would be very short-sighted if they did."

Almost all of the participants mention that building inspection has become stricter as well as that a good relationship with the inspectors is an advantage.

"Yes I have seen a difference; there are more insulations in new buildings now, especially in loft conversions. I do know most of the local Building Inspectors yes. Most of the small jobs that I do, I do on a Building Notice so I'll get the Inspector out at different stages and say this is what I want to do, is that ok? To be honest with you a lot of the times when I ask for an inspection if they're busy and they'll say 'we know you, just get on with it'."

Most notably our participants cannot see any governmental incentives for change; it is perceived to be more of a stick than a carrot approach. The cost of training is yet another prohibiting factor as is the time spent doing training (resulting in missing out on making money). One participant, who employs six staff, takes a refreshing approach; he installed a training room in their offices and tries to persuade his workers to have a look at instruction videos. Trade fairs are another way to keep up to date but this also means a considerable investment of time and money.

Irrespective of what they use computers for, participants have all been using them for a long time. Whether they operate from home or have an office elsewhere, the offices are rich in technology; computers, printers, (A3) scanners, faxes.

Maintaining their administration tends to tie them to the computer desk, and since they are juggling admin with going out to sites in the day-time, this often happens in the evening.

"The majority of the time I'm out, because I'm on site with the guys, so I do most of my paperwork and stuff on night which is a bit of a nightmare. Yes, most nights I'm sat here until ten."

Internet is indispensible as is wireless technology but in the countryside, where a good proportion of our interviewees reside, the internet often comes down to only a trickle.

"We've got a very slow Internet connection, we are right at the end of the line because the exchange is about five miles away."

The world has been embracing mobile technologies over the last two decades and builders are no exception. As can be expected, this revolves mostly around mobile phones and its infrastructure. However, the range of mobile appliances is certainly not restricted to these as cameras, calculators and electronic measurement tools are used regularly and they express the wish for being able to take (electronic) notes, use small form factor tablet-computers to access drawings, 
quotes etc. However, builders have been disappointed with the services that mobile infrastructures provide as they have to resort to bringing physical rather than digital artefacts, e.g. print-outs of plans, with them. In addition, particularly in the country side, there are the debilitating effects of poor signal strength.

"I think my phone is internet capable but to be honest the reception round here isn't very good cause you're up in the valleys. If it is a clear day and you haven't got any cloud cover, nothing works: Welcome to Wales."

Taking photographs, is one of the obvious and often utilised secondary benefits of a mobile phone and builders are knowledgeable users. Using photographs for referencing purposes, either for customers, building inspectors or employees, is common practice.

"The building inspector that we use because he knows that I'm not there to cut corners or rip anyone off, he is more than happy for me to, so I phone him up saying we are pouring the slab today do you want to come and have a look at it and he says have you got this have you got that take a few photos and then we can cross reference it afterwards."

Although we will present the findings of the HBXL software evaluation in full in the future, here we like to provide a taste of how interviewees express the considerable benefit and competitive advantage of using EstimatorXpress.

"Absolutely, absolutely, I'll show you the library of what they got on, on the package. The thing I like about this is: you can put your materials in and this works out all your quantities as well for what you need; so you go through the list and it tells you what sand and cement you need for the job and it will tell you, if you put a start date in it will tell you what dates you need to order them in on site. It's got down to the fact I need 7 cubic metres of skips and then it will automatically add on the landfill tax; put an allowance in there for petrol for using the disc-cutter where before that would just come out your profits. It's a very good tool for just making sure that you know exactly what's involved with the build."

\section{Discussion}

Against the background of the implementation of the Code for Sustainable Homes by 2016 , we conducted an in-depth interview study with SME builders. Most of the sample seems to have a good understanding of sustainable building methods and for some of them it is indeed part of their livelihood. However, all of them follow current building regulations thoroughly and value a good relationship with building inspectors.

Thus, at this stage of the research we (tentatively) conclude that SME builders are relatively positive about the current strict building regulations and for some the implementation of the $\mathrm{CSH}$ might be a way to combat the adverse effects of recession. Although there is a lack of affordable training schemes for these already struggling builders and there are few (if any) incentives to encourage a change in building practices.

As for technology use, those we interviewed are competent users of computers and mobile technologies. Although, it is debatable how widespread current technology use is amongst builders in general, based on this sample we should be cautious in automatically assuming that builders are starved of technology. 
The aim of our research program is to develop an easy to use integrated tool for SMEs to design sustainable buildings, incorporating CSH guidelines. Based on their current (and highly valued) use of HBXL's "estimating" software which allows them to gain intricate knowledge of all the different stages of the building process, it is likely that the planned software development has an educational role to play.

Focusing on mobile technologies, the impression that the interviewees create is that there is plenty of potential for using mobile technologies in the world of building but this is marred by poor reception, and, in spite of all the advertising hype, mobile appliances are not well suited to realise this potential. In short: there are great opportunities but there are still a lot of teething troubles.

Following on from the introduction of the iPad, a scaled down tablet computer, other manufacturers, e.g. Samsung and Microsoft, are following in its footsteps. However in spite of intensive advertising campaigns the industry has been disappointed by its sales figures [WEB ref 6] and it might take some time before a (late adopting) building industry starts to see value in using such appliances.

On a more general note, it is encouraging to see the use of psychological methods in gauging attitudes in the construction industry and it is understandable that researchers use the ubiquitous Likert scales even though this is a rather blunt tool.

However, the level of statistical analysis is disappointing as it seldom goes beyond listing percentages. Statistical analysis of a questionnaire benefits from testing similarities (using correlations and cluster analysis) and differences. The latter desirably through the analysis of variance (ANOVA) or at least (given the crudity of the scales) t-tests. In addition it is imperative to provide levels of statistical significance with accompanying degrees of freedom.

It is also good custom to start research with in-depth interviews (or even focus groups) where for a small sample important issues are elicited, leading to informed hypotheses. These hypotheses can then be tested through questionnaires targeting a wider population. The focus of another report is indeed the results of an on-line questionnaire to elicit input from a larger population of SME builders in order to test the hypotheses that were generated by the interview study.

\section{References}

1. Code for Sustainable Homes Technical Guide, published by Department for Communities and Local Government (2009)

2. Christiansson, P., K.B. Sorensen, M. Rodtness, M. Abrahamsen, L. Ostenfeld-Riemnann \& M. Alsdorf. User Driven Innovation in the Building Process. Tsinghua Science and Technology, 13(S1) (October 2008)

3. Dean, M. The Builder's role in delivering sustainable tall buildings. Structural Design Tall and Special Buildings 17, 869-880 (2008)

4. Dix, A., Finlay, J., Abowd, G., D., \& Beale, R. Human-computer interaction (3rd ed.). Harlow, England: Pearson Education Limited, 321 (2004)

5. Dixit, M.K., J.L. Fernández-Solís, S. Lavy, C.H. Culp, Identification of parameters for embodied energy measurement: A literature review, Energy and Buildings 42 1238-1247(2010) 
6. Downing, P., Understanding Consumer Attitudes to "Sustainable Community Infrastructure” "Research for the UK Green Building Council $\&$ the Zero Carbon Hub, undertaken by Icaro Consulting on behalf of the UK Green Building Council and the Zero Carbon Hub, and funded by the NHBC Foundation. (November 2009)

7. Gill, Z.M., M.J. Tierney; I.M. Pegga \& N. Allan, Low-energy dwellings: the contribution of behaviours to actual performance. Building Research \& Information 38(5), 491-508 (2010)

8. Gupta and Chandiwala, Understanding occupants: feedback techniques for large-scale low-carbon domestic refurbishments. Building Research \& Information (2010), 38(5), 530-548

9. Ornetzedera,M. \& H. Rohracherb, User-led innovations and participation processes: lessons from sustainable energy technologies, Energy Policy 34, 138-150 (2006)

10. Osmani, M. \& A. O'Reilly. Feasibility of zero carbon homes in England by 2016: A house builder's perspective. Building and Environment 44, 1917-1924 (2009)

\section{WEB references}

1. HBXL website: www.hbxl.co.uk

2. Integrated Environmental Solutions: http://www.iesve.com/UK-ROI, accessed in 2011

3. ISO 14000: http://www.iso14000-iso14001-environmentalmanagement.com/, accessed in 2010

4. Likert scales: http://intelligentmeasurement.wordpress.com/2007/11/20/likert-scalesurveys-best-practices/, accessed in 2010

5. Strategic Forum: http://www.strategicforum.org.uk/, accessed in 2010

6. Apple sales surge, but stocks slump as iPad figures disappoint. October 2010: http://www.digitaltrends.com/computing/apple-sales-surge-butstocks-slump-as-ipad-figures-disappoint/ 